\section{CHIAPAS 2019}

XV Congreso Latinoamericano de Patología de Construcción y

XVII Congreso de Control de Calidad en la Construcción CONPAT 2019, Vol. 1, CONTROL DE CALIDAD DOI: https://doi.org/10.21041/CONPAT2019/V1CC289CTE CONFERENCIA TEMÁTICA ESPECIALIZADA Tuxtla Gutiérrez, Chiapas, México Editado por: Francisco Alberto Alonso Farrera,

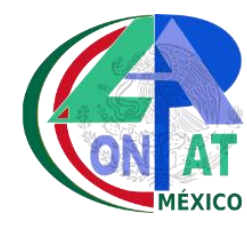
Francisco Javier Olguín Coca

\title{
SOLUCIONES CONSTRUCTIVAS EN MADERA. PROTECCIÓN Y DURABILIDAD LIGADA AL DISEÑO
}

\author{
M. $\operatorname{CONDE}^{1}$, S. MONTILLA ${ }^{2}$, B. ABAD ${ }^{1}$, J. A. TENORIO ${ }^{2}$ \\ ${ }^{1}$ Grupo de Investigación ERSAF. Escuela Técnica Superior de Ingeniería Agronómica y de Montes. \\ Universidad de Córdoba, Córdoba, España. \\ ${ }^{2}$ Grupo de Investigación Construcción Avanzada y Sostenible. Dep. Construcción. \\ Instituto de Ciencias de la Construcción Eduardo Torroja. CSIC, Madrid, España.
}

\begin{abstract}
RESUMEN
Actualmente la madera es un material de gran interés por sus valores asociados a la sostenibilidad, sin embargo, la incorrecta elección de los productos derivados de la madera provoca numerosas patologías que afectan a su durabilidad generando rechazo para su utilización en construcción. El Grupo Operativo Madera Construcción Sostenible trabaja en el proyecto "Herramienta inteligente para la selección de productos de madera destinados a la construcción" con el fin de facilitar la prescripción y la selección de productos más adecuada para cada uso. Se ha llevado a cabo una clasificación taxonómica, fichas de productos y valores ambientales de los mismos. También se proponen nuevas soluciones constructivas en base madera y una vinculación entre productos y soluciones constructivas que permitirá una selección adecuada de productos de madera, mejorando así su durabilidad.
\end{abstract}

Palabras clave: Productos de madera; sostenibilidad; soluciones constructivas; durabilidad; diseño. 


\section{INTRODUCCIÓN}

La madera en la construcción en España ha sido un referente como material tradicional hasta bien entrado el siglo XX y conforma por ello una buena parte del patrimonio construido. Los edificios con estructura de madera son altamente durables, aspecto que en sí es incuestionable y queda demostrado por el propio paso del tiempo. Este hecho en parte se debe a las técnicas constructivas utilizadas por nuestros antecesores, sencillas pero eficaces, que tienen en cuenta la protección de la madera desde el diseño de los edificios.

La madera hoy en día está cobrando un nuevo interés por parte de los arquitectos e ingenieros debido a los valores asociados a la sostenibilidad vista tanto desde el prisma ambiental como también económico y social. La madera además de ser un magnífico material de construcción es renovable y procede de montes certificados. Consumir madera en la construcción supone una forma clara de lucha contra el cambio climático.

La madera es un material tradicional pero también muy innovador. Los productos de construcción actuales en base madera poseen unas características que permiten a los sistemas constructivos proporcionar unas prestaciones muy superiores. La duda que surge está sin embargo relacionada con la durabilidad. Para garantizar la durabilidad de la madera ésta debe seleccionarse adecuadamente y estar convenientemente protegida.

Con el fin de facilitar la prescripción los autores están trabajando en el Proyecto GO Madera Construcción Sostenible. 'Herramienta inteligente para la selección de productos de madera destinados a la construcción' que haciendo uso de los sistemas más innovadores (BIM), proporcionará herramientas para facilitar el cumplimiento normativo mediante el desarrollo de un selector inteligente de productos, además de mostrar las excelencias ambientales. La comunicación mostrará las bases y los principales avances del trabajo en desarrollo.

\section{GRUPO OPERATIVO MADERA CONSTRUCCIÓN SOSTENIBLE}

\subsection{Descripción GO Madera Construcción Sostenible}

El grupo operativo Madera Construcción Sostenible está siendo coordinado por la Universidad de Córdoba, España, son miembros la Asociación para la Certificación Española Forestal PEFC (representante legal), INIA. Instituto Nacional de Investigación y Tecnología Agraria y Alimentaria; IETCC. CSIC. Instituto Eduardo Torroja de ciencias de la construcción de la Agencia Estatal Consejo Superior de Investigaciones Científicas; Cupa Innovación S.L.U.; THERMOCHIP S.L.; BJD S.L.; BALIDEA S.L.; y AENOR Internacional S.A.U. Como miembros colaboradores participan MADERIA. Sociedad Española de la Madera; COSE. Confederación de organizaciones de selvicultores de España; y MADERAS ABAD S.L. El proyecto está financiado por el Programa Nacional de Desarrollo Rural 2014-2020, a través del Fondo Europeo Agrícola de Desarrollo Rural FEADER y el Ministerio de Agricultura, Pesca y Alimentación.

El proyecto pondrá a disposición de los prescriptores y otros agentes del sector de la construcción, un selector de productos de madera. Uno de los objetivos es fomentar un mayor conocimiento del material y mejorar su durabilidad a través de la correcta utilización de los productos de madera.

\subsection{Objetivos}

El objetivo general del proyecto es posicionar en el mercado los productos de madera para la construcción por sus valores técnicos y ambientales impulsando la cadena de valor desde el monte hasta el consumidor final.

Como objetivos específicos se marcan los siguientes:

- Proporcionar información y datos técnicos fiables sobre productos de madera para facilitar la prescripción. 
- Demostrar mediante información ambiental la excelencia de la madera como material de construcción en relación con los valores asociados a la sostenibilidad.

- Proporcionar una herramienta para la transmisión de la información de las características técnicas y ambientales de productos de madera para la construcción.

\subsection{Trabajos realizados}

\subsubsection{Productos de madera}

Las actividades se dirigieron a simplificar la elección de la madera y los productos derivados de esta más adecuados para cada uso en construcción. Es totalmente objetivo afirmar que una buena elección de un producto de madera contribuye a aumentar su durabilidad y reduce la necesidad de protección ante los factores externos. Sin embargo, se ha detectado que esta tarea no es sencilla para los técnicos de la construcción debido a la diversidad de usos y de productos de madera presentes en el mercado.

Por otra parte, existe, al menos en España, un gran rechazo a elegir productos de madera para la construcción entre los prescriptores y los potenciales usuarios finales, debido a las numerosas patologías que, asociadas a una mala elección de los productos, pueden producirse. A pesar de que hay numerosas evidencias que demuestran lo contrario, el uso inapropiado de los productos de madera en la construcción ha forjado la imagen de la madera como un material de durabilidad dudosa. Proporcionar las herramientas para una correcta elección ha sido la clave técnica del desarrollo de las primeras actividades realizadas.

Tras un estudio exhaustivo de los productos de madera utilizados en construcción se ve que no es sencilla ni inmediata su clasificación. La división por familias puede atender a múltiples criterios. Lo óptimo sería establecer un sistema de ordenación y clasificación flexible para ser implementado fácilmente en la herramienta inteligente a desarrollar en el proyecto.

\section{Selección de productos tipo derivados de la madera}

Se han identificado los productos de construcción más utilizados en España, a la vez que se han planteado las posibles agrupaciones en familias de productos. La clasificación final elegida atiende al criterio de ordenar los productos según el grado de desintegración que sufre la madera en su fabricación. Las familias de productos de madera seleccionadas son las siguientes:

1. Madera aserrada estructural de sección rectangular

2. Madera laminada encolada (MLE)

3. Tableros de madera maciza multicapa (SWP)

4. Tableros contralaminados de madera maciza (CLT)

5. Tableros contrachapados

6. Productos microlaminados LVL

7. Tablero OSB

8. Tablero de partículas

9. Tablero de fibras densidad media MDF

10. Tableros sándwich

Para la identificación y selección de productos tipo se han empleado las definiciones de clases de uso (CU) y clases de servicio (CS), ya que no solo es importante para prever la durabilidad biológica y mecánica del material en función de las condiciones de humedad y temperatura ambientales en las que esté trabajando (prestando servicio) un producto, sino también para definir las clases técnicas de cada una de las familias de productos.

La clase de uso es un término que hace referencia al riesgo de ataque por organismos xilófagos, que tiene una madera trabajando en unas condiciones determinadas de humedad. La norma UNE-EN 335, en debate en el Comité Europeo de Normalización, define 5 clases de uso, incluyendo una subdivisión de una de las clases en dos más. Esta norma contempla el riesgo de ataque fundamentalmente por hongos. 
La clase de servicio está relacionada con el comportamiento mecánico del material, tanto de su resistencia como su elasticidad, según las condiciones ambientales en las que el producto esté trabajando en servicio. (Fernández-Golfín et al., 2011). Además, este concepto adquiere especial importancia en aquellos productos que incorporen adhesivos en su fabricación, ya que la clase de servicio está relacionada con la integridad y seguridad estructural, dado que todos los adhesivos tienen que cumplir unas determinadas exigencias para asegurar la integridad de la pieza y por tanto la permanencia del producto. El Código Técnico de la Edificación y la norma UNE-ENV 1995-1 establecen tres clases de servicio para las distintas condiciones higrométricas.

Además del concepto de clase técnica, para cada una de las familias de productos, se han empleado diferentes criterios para el establecimiento de productos tipo tales como las clases resistentes, los espesores o las especies de madera utilizada.

En esta primera etapa se han seleccionado y analizado un total de 113 productos tipo de madera.

\section{Realización de un sistema taxonómico de clasificación}

Los trabajos han consistido en el análisis de un gran número de clasificaciones de productos de madera atendiendo a distintos criterios para proponer un sistema taxonómico de clasificación. Por otra parte, se ha estudiado el conjunto de productos seleccionado con el objetivo de comprobar cuáles pueden formar parte de las soluciones constructivas.

La taxonomía tiene la importancia de clasificar mediante un sistema jerárquico y sistemático los productos. La simplicidad es clave para su utilización, el orden tiene importancia para agilizar las búsquedas.

La conclusión del estudio establece la siguiente clasificación taxonómica:

- Dominio: Productos

- Reino: Productos de madera y otros productos

- División: Productos de madera para uso general y para uso en la construcción

- Clase: Productos en forma de perfil o en forma de tablero o panel.

- Orden: Producto con uso estructural o con uso no estructural

- Género: Clase técnica definida por la clase de uso y clase de servicio.

- Categoría: Funciones u otros requisitos específicos.

Como se puede observar no aparece el término familia para evitar confusiones con las familias anteriormente definidas.

Recopilación de características técnicas de las familias de productos y elaboración de fichas tipo Para definir los productos clasificados, se ha llevado a cabo un análisis y recopilación de las características (mecánicas, comportamiento al fuego, durabilidad, etc.) más relevantes de la madera y sus productos derivados, de cara a su caracterización para su uso en construcción.

A partir de las familias de productos de madera seleccionadas se han estudiado y recopilado las características técnicas de cada una de las familias. Se ha trabajado con los aspectos más relevantes a la hora de su utilización como producto de construcción (dimensiones, tolerancias, características y propiedades), así como su caracterización para su vinculación con las soluciones constructivas, normas de referencia, clases de uso y servicio, etc.

Toda esta información se recoge en un conjunto de fichas tipo elaboradas para los productos seleccionados de las familias establecidas. Dada la gran cantidad y variedad de productos del mercado sólo se ha podido abarcar una parte de los mismos.

\subsubsection{Soluciones constructivas}

El diseño de nuevas soluciones constructivas en base madera pretende completar una labor iniciada hace más de diez años con el Catálogo de Elementos Constructivos del Código Técnico de la Edificación (CTE) del Ministerio de Fomento de España, para ampliar el abanico de soluciones 
constructivas, actualizar el contenido con nuevas soluciones más industrializadas, relacionar mejor soluciones y productos y mostrarlo en el formato actual mediante modelos BIM.

El punto de partida ha sido el análisis del Catálogo de Elementos Constructivos de 2010. Se ha hecho un estudio exhaustivo de soluciones posibles, tratando de generalizar las soluciones viables con madera, obteniéndose una variedad compleja que supera las expectativas iniciales del proyecto. En el catálogo de elementos constructivos existían soluciones que contienen productos de madera, fundamentalmente tableros, junto con otros materiales, aunque también alguna solución en base madera, en concreto una de cubierta, una de fachada y una partición.

Tras el estudio realizado, el total de soluciones susceptible de ser industrializadas propuestas, desarrolladas y descritas es de: 40 soluciones de cubierta, 11 de fachada, 8 particiones y 24 forjados tipo. Estas soluciones podrían incrementarse si se tienen en consideración en algunos casos de forma independiente las soluciones con trasdosado.

Dada la gran labor que conlleva el estudio pormenorizado de todas las soluciones propuestas y los recursos disponibles en el proyecto, se ha comenzado por evaluar 3 soluciones nuevas de cubierta, 5 fachadas, 3 particiones y 2 forjados tipo.

\section{Verificación de prestaciones frente a las exigencias reglamentarias del CTE}

Para las soluciones seleccionadas se han analizado las prestaciones de comportamiento frente a la humedad, frente al fuego, acústica y térmica. Se ha comprobado la bondad de las soluciones sobre todo en los aspectos relacionados con la durabilidad.

Se ha establecido la forma de mostrar los resultados para facilitar el desarrollo de modelos BIM. En este punto también ha sido necesario estudiar la forma de expresar los resultados para que puedan ser visualizados de forma ágil por la herramienta inteligente.

Se hace notar que las prestaciones a incluir en las soluciones provienen de estimaciones conservadoras. Sería de gran interés hacer una campaña de ensayos de aislamiento acústico para algunas soluciones a fin de optimizarlas. Esta actividad por coste excede en mucho el alcance del proyecto, pero sería sin duda de gran interés.

\subsubsection{Vinculación de las soluciones constructivas con los productos de la clasificación taxonómica. Selector.}

Se han estudiado distintas opciones para la vinculación de las soluciones constructivas y los productos de construcción. Inicialmente se trató de hacer una correlación entre la taxonomía propuesta y la vinculación según la metodología propuesta en el VII Congreso Forestal Español (Conde et al., 2017). Esto suponía la necesidad de establecer nuevos taxones a fin de incorporar los términos de adecuación al uso. Finalmente se ha establecido la correlación mediante la codificación de los productos que forman parte de las soluciones constructivas. El problema reside en que la codificación no es biunívoca, ya que a una solución pueden corresponderle varios productos y viceversa.

De esta forma, el código se establece a nivel de solución y los productos se vinculan a las soluciones adoptando dicho código. El sistema de codificación es escalable.

\section{Desarrollo de modelos BIM}

Se han establecido las bases para el desarrollo de los modelos BIM. En este momento se están empezando a generar los primeros modelos de las soluciones.

Aunque pueda parecer que todos los modelos son iguales y compatibles no es cierto. Para establecer las bases se ha desarrollado un estado del arte, se han analizado las especificaciones a requerir a los objetos para cumplir con los estándares de calidad, conectarse con objetos genéricos y de fabricantes y asegurar que la colaboración e interoperabilidad con las distintas plataformas BIM se cumpla. Las normas que se siguen son estrictamente las europeas OPENBIM. 


\subsubsection{Valores ambientales}

Es una realidad la baja implantación en España de las Declaraciones Ambientales de Producto en el sector de la construcción, aunque en el resto de Europa esta actividad se encuentra mucho más desarrollada. En el sector de la madera existen fabricantes muy involucrados, aunque aún no está generalizada su utilización.

Sin embargo, los sellos ambientales sí tienen una mayor aceptación, sobre todo en la parte de la cadena más cercana al sector forestal. La implantación de los sistemas de certificación forestal en el monte y cadena de custodia en las industrias de la madera, ha hecho al sector forestal pionero en el establecimiento de indicadores para evaluar y garantizar la sostenibilidad en sus actividades, asegurando un uso sostenible de los recursos y contribuyendo a la conservación y mejora de sus masas forestales.

En el proyecto del Grupo Operativo se ha llevado a cabo un estudio de las familias de productos de madera más importantes desde los aspectos ambientales, donde se han analizado los procesos que intervienen en la fabricación, desde un punto de vista genérico.

Mediante la metodología del Análisis de Ciclo de Vida se han evaluado un conjunto de productos procedentes de la industria de la primera transformación, con el fin de obtener los datos de inventario genéricos de productos tipo de las distintas familias de productos. Se han estudiado los procesos de fabricación y se ha realizado una evaluación del consumo de recursos, materias primas y energía. Las industrias analizadas corresponden fundamentalmente a aserraderos y fábricas de tableros asentadas en España.

\section{RESULTADOS}

\subsection{Soluciones constructivas: diseño y prestaciones.}

En las siguientes tablas 1, 2, 3 y 4 se recogen las soluciones constructivas diseñadas en el proyecto y los códigos utilizados para su nomenclatura. En total se han propuesto 8 Forjados, 40 Cubiertas, 11 Fachadas y 24 Particiones verticales.

Tabla 1. Clasificación de soluciones constructivas para forjados.

\begin{tabular}{|c|c|c|c|c|}
\hline \multirow{8}{*}{ FORJADO } & \multirow[t]{2}{*}{ ENTRAMADO } & $\begin{array}{c}\text { ESTRUCTURA } \\
\text { VISTA }\end{array}$ & & FO ENT-EV \\
\hline & & FALSO TECHO & & FO ENT-FT \\
\hline & \multirow{4}{*}{ CLT } & \multirow{2}{*}{ SIMPLE } & Trasdosado & FO CLT-T \\
\hline & & & No trasdosado & FO CLT-NT \\
\hline & & \multirow{2}{*}{ DOBLE } & Trasdosado & FO 2CLT-T \\
\hline & & & No trasdosado & FO 2CLT-NT \\
\hline & \multirow{2}{*}{ MIXTO } & $\begin{array}{c}\text { ESTRUCTURA } \\
\text { VISTA }\end{array}$ & & FO MX-EV \\
\hline & & FALSO TECHO & & FO MX-FT \\
\hline
\end{tabular}


Tabla 2. Clasificación de soluciones constructivas para cubiertas.

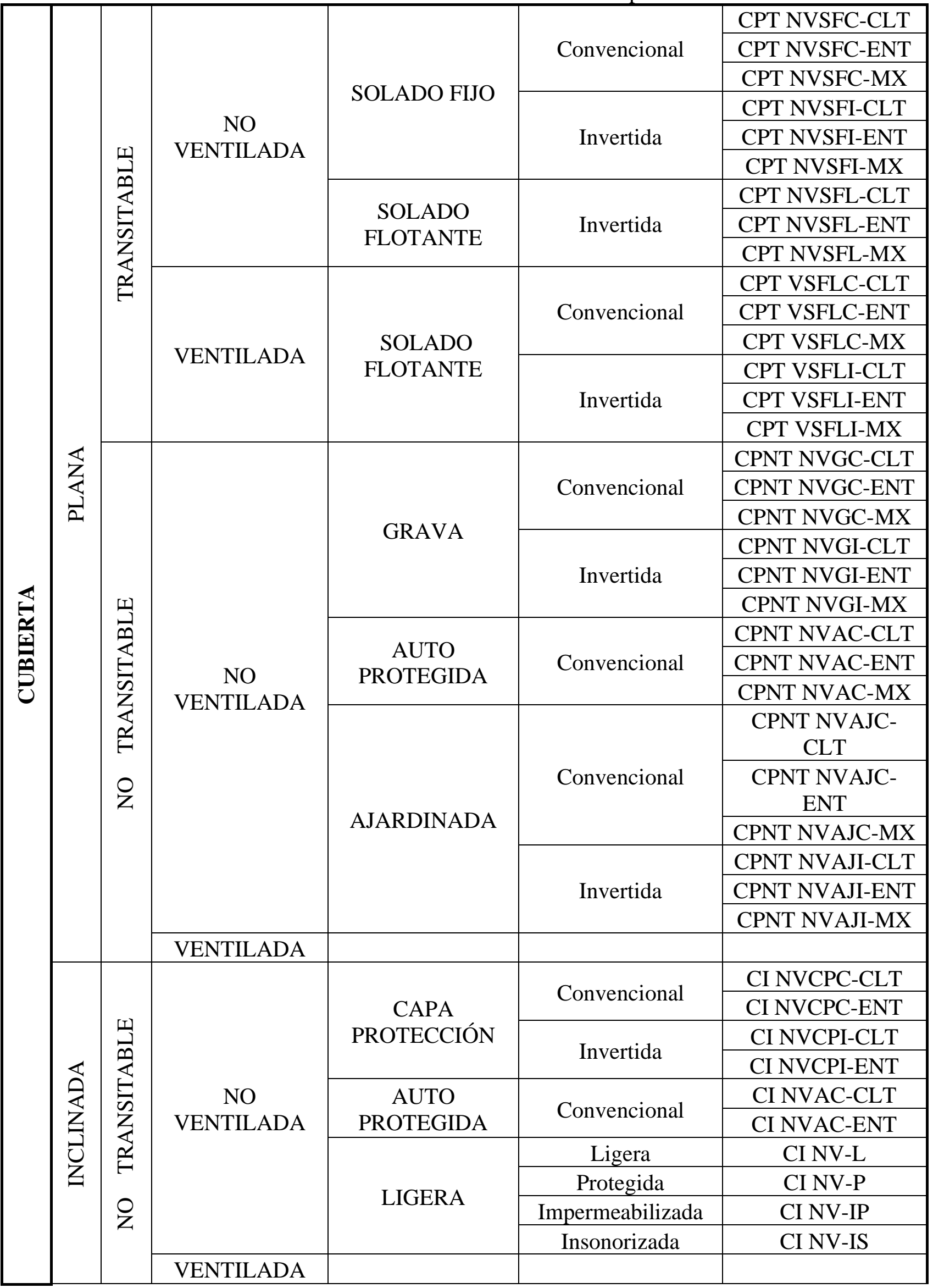


Tabla 3. Clasificación de soluciones constructivas para fachadas.

\begin{tabular}{|c|c|c|c|c|}
\hline \multirow{12}{*}{ FACHADA } & \multirow{4}{*}{ PESADA } & \multirow{2}{*}{ ENTRAMADO } & SATE & FAP ENT-SATE \\
\hline & & & CÁMARA VENTILADA & FAP ENT-CV \\
\hline & & \multirow[b]{2}{*}{ CLT } & SATE & FAP CLT-SATE \\
\hline & & & CÁMARA VENTILADA & FAP CLT-CV \\
\hline & \multirow{6}{*}{ LIGERA } & \multirow{2}{*}{ ENTRAMADO } & SATE & FAL ENT-SATE \\
\hline & & & CÁMARA VENTILADA & FAL ENT-CV \\
\hline & & \multirow{4}{*}{ PANELES } & SIMPLE & FAL $\mathrm{P}$ \\
\hline & & & PROTEGIDA & FAL P-P \\
\hline & & & IMPERMEABILIZADA & FAL P-IP \\
\hline & & & INSONORIZADA & FAL P-IS \\
\hline & \multirow{2}{*}{ MIXTA } & & SATE & FAX-SATE \\
\hline & & & CÁMARA VENTILADA & FAX-CV \\
\hline
\end{tabular}

Tabla 4. Clasificación de soluciones constructivas para particiones verticales.

\begin{tabular}{|c|c|c|c|c|c|c|}
\hline \multirow{25}{*}{ 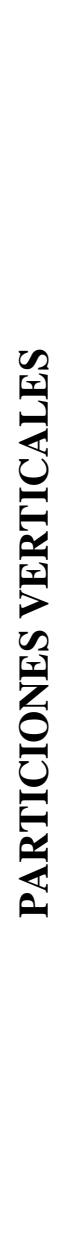 } & \multirow{9}{*}{ 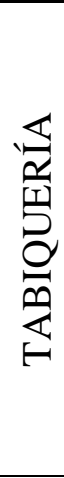 } & \multirow{5}{*}{$\stackrel{\ominus}{\ominus}$} & \multirow{3}{*}{ TRASDOSADO } & SIMPLE & & PTAB CLT-T \\
\hline & & & & DOBLE & & PTAB CLT-2T \\
\hline & & & & TRIPLE & & PTAB CLT-3T \\
\hline & & & \multirow{2}{*}{$\begin{array}{c}\text { NO } \\
\text { TRASDOSADO }\end{array}$} & SIMPLE & & PTAB CLT-NT \\
\hline & & & & DOBLE & & PTAB 2CLT-NT \\
\hline & & \multirow{4}{*}{ 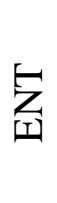 } & & SIMPLE & & PTAB ENT-S \\
\hline & & & & DOBLE & & PTAB ENT-D \\
\hline & & & & TRIPLE & & PTAB ENT-T \\
\hline & & & & RASTREL & & PTAB ENT-R \\
\hline & \multirow{16}{*}{$\stackrel{\square}{\sim r}$} & \multirow{10}{*}{$\stackrel{H}{\mathcal{U}}$} & \multirow{5}{*}{ CLT SIMPLE } & \multirow{2}{*}{ NO TRASD. } & 2 ISA & PSEU CLT-NT2ISA \\
\hline & & & & & $2 \mathrm{AT}+\mathrm{ISA}$ & PSEU CLT-NT2AT + ISA \\
\hline & & & & \multicolumn{2}{|c|}{ TRASD. SIMPLE } & \\
\hline & & & & DOBLE & SIMPLE & PSEU CLT-2TS \\
\hline & & & & TRASD. & DOBLE & PSEU CLT-2TD \\
\hline & & & \multirow{5}{*}{ CLT DOBLE } & \multirow{3}{*}{ NO TRASD. } & AT & PSEU 2CLT-NTAT \\
\hline & & & & & ISA & PSEU 2CLT-NTISA \\
\hline & & & & & $\mathrm{AT}+\mathrm{CA}$ & PSEU 2CLT-NTAT + CA \\
\hline & & & & \multirow{2}{*}{ TRASD. } & SIMPLE & PSEU 2CLT-TS \\
\hline & & & & & DOBLE & PSEU 2CLT-TD \\
\hline & & \multirow{6}{*}{ 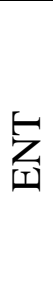 } & SIMPLE & DOBLE AT & & PSEU ENT-2AT \\
\hline & & & \multirow{5}{*}{ DOBLE } & \multirow{2}{*}{$\mathrm{CA}$} & ARRIOST. & PSEU 2ENT-CA Arrio \\
\hline & & & & & SIN ARRIOST & PSEU 2ENT-CAS Arrio \\
\hline & & & & \multirow{3}{*}{ AT } & SIMPLE (T) & PSEU 2ENT-ATS \\
\hline & & & & & DOBLE (2T) & PSEU 2ENT-ATD \\
\hline & & & & & TRIPLE (3T) & PSEU 2ENT-ATT \\
\hline
\end{tabular}

Cómo se ha dicho en el apartado 2.3.2, debido a que labor de caracterizar todas las soluciones supera en gran medida lo detallado en el proyecto, se ha comenzado por evaluar 3 soluciones nuevas de cubierta, 5 de fachada, 3 de partición y 2 forjados tipo.

En la tabla 5 se incluye la propuesta de soluciones seleccionadas. Nótese que en la tabla figuran más soluciones tipo de las señaladas anteriormente ya que son realmente variedades del mismo tipo (algunas de las soluciones se duplican en función de si presentan o no trasdosado). 
Tabla 5. Recopilación soluciones constructivas.

\begin{tabular}{|c|c|c|}
\hline Tipo & NOMBRE & CLASIFICACIÓN \\
\hline \multirow{6}{*}{ Cubiertas } & CPT NVSFC-CLT & $\begin{array}{l}\text { Cubierta , Plana, Transitable, No ventilada, Solado Fijo, } \\
\text { Convencional, CLT }\end{array}$ \\
\hline & CPT NVSFC-CLT t & $\begin{array}{l}\text { Cubierta , Plana, Transitable, No ventilada, Solado Fijo, } \\
\text { Convencional, CLT, trasdosado }\end{array}$ \\
\hline & CPT NVSFC-ENT & $\begin{array}{l}\text { Cubierta , Plana, Transitable, No ventilada, Solado Fijo, } \\
\text { Convencional, Entramado }\end{array}$ \\
\hline & CPT NVSFC-ENT $\mathrm{t}$ & $\begin{array}{l}\text { Cubierta , Plana, Transitable, No ventilada, Solado Fijo, } \\
\text { Convencional, Entramado, trasdosado }\end{array}$ \\
\hline & CPT NVSFC-MX & $\begin{array}{l}\text { Cubierta , Plana, Transitable, No ventilada, Solado Fijo, } \\
\text { Convencional, Mixto }\end{array}$ \\
\hline & CPT NVSFC-MX t & $\begin{array}{l}\text { Cubierta , Plana, Transitable, No ventilada, Solado Fijo, } \\
\text { Convencional, Mixto, trasdosado }\end{array}$ \\
\hline \multirow{9}{*}{ Fachadas } & FAP ENT - CV & Fachada, Pesada, Entramado, Cámara ventilada \\
\hline & FAP ENT - CV t & Fachada, Pesada, Entramado, Cámara ventilada, trasdosado \\
\hline & FAP CLT - SATE & Fachada, Pesada, CLT, SATE \\
\hline & FAP CLT - SATE $\mathrm{t}$ & Fachada, Pesada, CLT, SATE, trasdosado \\
\hline & FAL ENT - SATE & Fachada, Ligera, Entramado, SATE \\
\hline & FAL ENT - SATE $\mathrm{t}$ & Fachada, Ligera, Entramado, SATE, trasdosado \\
\hline & FAL ENT - Cva & $\begin{array}{l}\text { Fachada, Ligera, Entramado, Cámara ventilada, Protección } \\
\text { discontinua }\end{array}$ \\
\hline & FAL ENT - Cva t & $\begin{array}{l}\text { Fachada, Ligera, Entramado, Cámara ventilada, Protección } \\
\text { discontinua, trasdosado }\end{array}$ \\
\hline & FAL PS - IS t & Fachada, Ligera, Panel Sándwich, Insonorizada, trasdosado \\
\hline \multirow{4}{*}{ Particiones } & P TAB ENT - S & Particiones, Tabiquería, Entramado, Simple \\
\hline & P TAB ENT - S t & Particiones, Tabiquería, Entramado, Simple, trasdosado \\
\hline & P SEU CLT - 2T D & Particiones, Separación Entre Usuarios, CLT, Doble Trasdosado \\
\hline & P SEU 2CLT - T S & $\begin{array}{l}\text { Particiones, Separación Entre Usuarios, Doble CLT, Trasdosado, } \\
\text { Simple }\end{array}$ \\
\hline \multirow{3}{*}{ Forjados } & FO ENT - FT & Forjado, Entramado, Falso techo (Estructura oculta) \\
\hline & FO ENT - FT t & Forjado, Entramado, Falso techo (Estructura oculta), Trasdosado \\
\hline & FO CLT - T & Forjado, CLT, Trasdosado \\
\hline
\end{tabular}

A continuación, se muestra a modo de ejemplo del trabajo realizado, la ficha de dos de las soluciones constructivas analizadas. Todas las fichas incluyen una descripción de la solución, descripción de los materiales que componen cada elemento constructivo y los valores de prestaciones térmicas, acústicas y de salubridad (esta última no incluida para forjados y particiones). 
Tabla 6. Descripción de la solución constructiva CPT NVSFC-ENT. Elementos que la componen. CPT NVSFC-ENT

Cubierta, Plana, Transitable, No ventilada, Solado Fijo, Convencional, Entramado

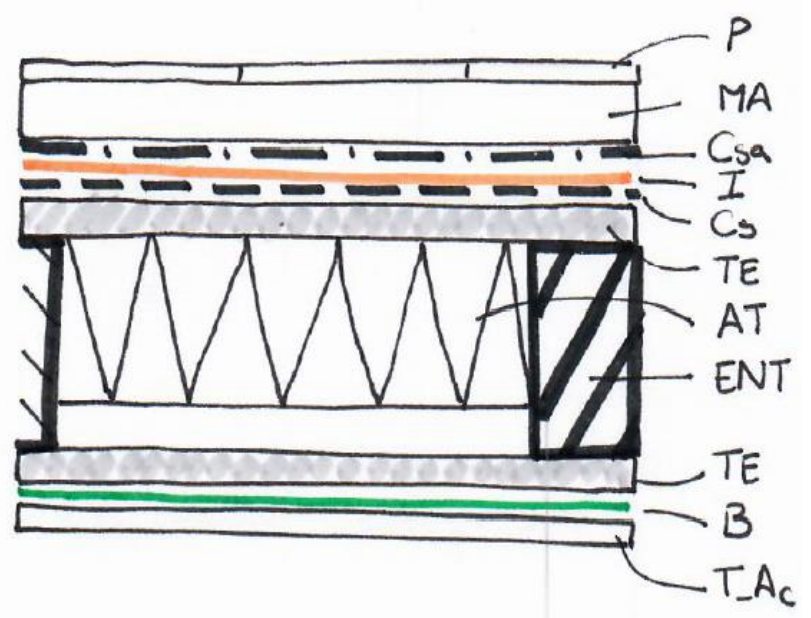

P: $\quad$ Capa protección solado fijo

MA: $\quad$ Material de agarre o nivelación (mortero, lecho de arena...)

Csa: Capa separadora antipunzonante bajo protección

I: $\quad$ Capa impermeabilización

Cs: $\quad$ Capa separadora

TE: Tablero estructural madera (cara fría)

AT: Aislante

ENT: Soporte estructural entramado vigas de madera

TE: $\quad$ Tablero estructural madera (cara caliente)

B: Barrera contra el vapor en cubierta convencional

T_Ac: Tablero acabado

Tabla 7. Caracterización de materiales que forman la solución constructiva CPT NVSFC-ENT.

\begin{tabular}{|c|c|c|c|c|c|c|}
\hline \multicolumn{2}{|c|}{ Material } & $\begin{array}{c}\text { Espesor } \\
(\mathbf{m m})\end{array}$ & $\begin{array}{c}\boldsymbol{\rho} \\
(\mathbf{k g} / \mathbf{m 3})\end{array}$ & $\begin{array}{c}\boldsymbol{\lambda} \\
(\mathbf{W} / \mathbf{m} . \mathbf{K})\end{array}$ & $\begin{array}{c}\text { Cp } \\
(\mathbf{J} / \mathbf{k g} . \mathbf{K})\end{array}$ & $\begin{array}{c}\boldsymbol{\mu} \\
\text { (adim.) }\end{array}$ \\
\hline P1 & Baldosa & 6 & 2000 & 1 & 800 & 30 \\
\hline MA & Mortero Cemento & 50 & 2000 & 1,8 & 1000 & 10 \\
\hline Cs1 & $\begin{array}{c}\text { Geotextil } \\
\text { antipunzonante } \\
\text { (FIELTRO) }\end{array}$ & 1,1 & 120 & 0,05 & 130 & 15 \\
\hline I1 & $\begin{array}{c}\text { Material Bituminoso } \\
\text { Monocapa }\end{array}$ & 5 & 1500 & 0,475 & 1000 & 50000 \\
\hline AT1 & $\begin{array}{c}\text { Tablero aislante fibra } \\
\text { madera }\end{array}$ & 120 & 170 & 0,04 & - & 1 \\
\hline AT2 & Lana de roca & 120 & 100 & 0,035 & - & 1 \\
\hline B & $\begin{array}{c}\text { Lámina polietileno baja } \\
\text { densidad }\end{array}$ & 0,2 & 920 & 0,33 & 2200 & 100000 \\
\hline ENT1 & Madera maciza & 140 & 550 & 0,18 & 1600 & 20 \\
\hline ENT2 & Madera maciza & 160 & 540 & 0,13 & - & - \\
\hline TE1 & Tablero Estructural & 40 & 650 & 0,13 & 1700 & 30 \\
\hline T_Ac1 & Tablero yeso & 15 & 900 & 0,25 & - & 280 \\
\hline T_Ac2 & $\begin{array}{c}\text { Tablero acabado } \\
\text { madera }\end{array}$ & 20 & 550 & 0,156 & - & 20 \\
\hline
\end{tabular}


Tabla 8. Prestaciones según el Código Técnico de la Edificación, para la solución constructiva CPT NVSFC-ENT.

\begin{tabular}{|c|c|c|c|c|c|c|c|}
\hline \multicolumn{8}{|c|}{ Prestaciones solución constructiva } \\
\hline & \multirow[b]{2}{*}{$\begin{array}{c}\text { Espesor } \\
\text { total }\end{array}$} & \multirow[b]{2}{*}{ REI } & \multirow{2}{*}{$\begin{array}{c}\text { HE } \\
\mathbf{U} \\
{[\mathrm{W} /(\mathrm{m} 2 / \mathrm{K})]}\end{array}$} & \multicolumn{4}{|c|}{ HR } \\
\hline & & & & $\underset{(\mathrm{kg} / \mathrm{m} 2)}{\mathbf{m}}$ & $\begin{array}{c}\text { RA } \\
(\mathbf{d B A})\end{array}$ & $\begin{array}{l}\text { RATR } \\
\text { (dBA) }\end{array}$ & $\begin{array}{l}\text { RW } \\
\text { (dB) }\end{array}$ \\
\hline $\begin{array}{c}\text { Variante 1: AT, } \\
\text { ENT1 }\end{array}$ & $298,2+\mathrm{E}_{\mathrm{AT}}$ & - & $1 /\left(1,672+\mathrm{R}_{\mathrm{AT}}\right)$ & 243,62 & 49 & 44 & - \\
\hline $\begin{array}{c}\text { Variante 2: AT, } \\
\text { ENT2 }\end{array}$ & $323,2+\mathrm{E}_{\mathrm{AT}}$ & - & $1 /\left(1,96+\mathrm{R}_{\mathrm{AT}}\right)$ & 261,16 & 48 & 43 & - \\
\hline
\end{tabular}

Tabla 9. Descripción de la solución constructiva FAP CLT - CVt y elementos que la componen.

\begin{tabular}{|l|l|l|l|}
\hline FAP CLT - CVt \\
\hline Fachada, Pesada, CLT, Cámara ventilada, trasdosado \\
\hline
\end{tabular}

Tabla 10. Caracterización de materiales que forman la solución constructiva FAP CLT - CVt.

\begin{tabular}{|c|c|c|c|c|c|c|}
\hline \multicolumn{7}{|c|}{ Caracterización de materiales } \\
\hline & Material & $\begin{array}{c}\text { Espesor } \\
(\mathbf{m m})\end{array}$ & $\begin{array}{c}\boldsymbol{\rho} \\
(\mathbf{k g} / \mathbf{m} 3)\end{array}$ & $\begin{array}{c}\boldsymbol{\lambda} \\
(\mathbf{W} / \mathbf{m} . \mathbf{K})\end{array}$ & $\begin{array}{c}\mathbf{C p} \\
(\mathbf{J} / \mathbf{k g . K})\end{array}$ & $\begin{array}{c}\boldsymbol{\mu} \\
(\mathbf{a d i m} .)\end{array}$ \\
\hline P5 & $\begin{array}{c}\text { Protección fachada: } \\
\text { paneles madera }\end{array}$ & 30 & 550 & 0,15 & - & - \\
\hline Pe1 & Perfilería metálica & 40 & 350 & 0,13 & - & 50 \\
\hline Pe2 & $\begin{array}{c}\text { Perfilería rastreles } \\
\text { madera }\end{array}$ & 40 & 450 & 0,15 & 1600 & 20 \\
\hline TE 1 & Tablero Estructural & 40 & 650 & 0,13 & 1700 & 30 \\
\hline AT1 & $\begin{array}{c}\text { Tablero aislante fibra } \\
\text { madera }\end{array}$ & 120 & 170 & 0,04 & - & 1 \\
\hline AT2 & Lana de roca & 120 & 100 & 0,035 & - & 1 \\
\hline CLT1 & Madera contralaminada & 100 & 500 & 0,13 & - & 50 \\
\hline CLT2 & Madera contralaminada & 120 & 500 & 0,13 & - & 50 \\
\hline T_Ac1 & Tablero yeso & 15 & 900 & 0,25 & - & 280 \\
\hline T_Ac2 & Tablero acabado & 30 & 550 & 0,156 & - & - \\
\hline
\end{tabular}


Tabla 11. Prestaciones constructivas según el Código Técnico de la Edificación, para la solución constructiva FAP CLT - CVt.

\begin{tabular}{|c|c|c|c|c|c|c|c|}
\hline \multicolumn{8}{|c|}{ Prestaciones solución constructiva } \\
\hline & \multirow{2}{*}{$\begin{array}{c}\text { Espesor } \\
\text { total }\end{array}$} & \multirow[b]{2}{*}{ REI } & \multirow{2}{*}{$\begin{array}{c}\mathrm{HE} \\
\mathrm{U} \\
{[\mathrm{W} /(\mathrm{m} 2 / \mathrm{K})]}\end{array}$} & \multicolumn{3}{|c|}{ HR } & \multirow{2}{*}{$\frac{\text { HS }}{\text { GI }}$} \\
\hline & & & & $\underset{(\mathrm{kg} / \mathrm{m} 2)}{\mathbf{m}}$ & $\begin{array}{c}\mathbf{R}_{\mathbf{A}} \\
(\mathbf{d B A})\end{array}$ & $\begin{array}{c}\mathbf{R}_{\text {ATR }} \\
(\text { dBA })\end{array}$ & \\
\hline $\begin{array}{c}\text { Variante 1: AT, } \\
\text { CLT1, PE1, } \\
\text { AT4,T_Ac1 }\end{array}$ & $305+\mathrm{E}_{\mathrm{AT}}$ & - & $1 /\left(\mathrm{R}+\mathrm{R}_{\mathrm{AT}}\right)$ & 198,40 & 48 & 42 & 4 \\
\hline $\begin{array}{c}\text { Variante 2: AT, } \\
\text { CLT2, PE2, } \\
\text { AT5,T_Ac2 }\end{array}$ & $340+\mathrm{E}_{\mathrm{AT}}$ & - & $1 /\left(\mathrm{R}+\mathrm{R}_{\mathrm{AT}}\right)$ & 166 & 48 & 42 & 4 \\
\hline
\end{tabular}

\subsection{Vinculación entre productos y soluciones constructivas}

Los productos que pueden componer cada elemento constructivo se vinculan con las soluciones a través de códigos. La variable que más influye para la selección de los productos de madera es la clase de uso y servicio para la que son aptos o no cada producto según las condiciones higrométricas en las que estará expuesto. En cualquier caso, las condiciones pueden modificarse en función de condicionamientos del proyecto.

Tabla 12. Vinculación de productos de madera para cada elemento constructivo de madera de la solución constructiva CPT NVSFC-ENT.

\begin{tabular}{|c|c|c|c|c|}
\hline $\begin{array}{c}\text { ELEMENTO } \\
\text { CONSTRUCTIVO }\end{array}$ & PRODUCTOS & $\begin{array}{l}\text { CLASE } \\
\text { DE USO }\end{array}$ & $\begin{array}{l}\text { CLASE DE } \\
\text { SERVICIO }\end{array}$ & NORMA \\
\hline \multirow{5}{*}{$\begin{array}{l}\text { Tablero estructural } \\
\text { (cara fría) }\end{array}$} & $\begin{array}{l}\text { Tablero de partículas Clase } \\
\text { Técnica P5 (Ambiente } \\
\text { húmedo). }\end{array}$ & 2 & 2 & UNE-EN 312:2010 \\
\hline & $\begin{array}{l}\text { Tablero OSB Clase Técnica } \\
\text { OSB/3 (Ambiente húmedo). }\end{array}$ & 2 & 2 & UNE-EN 300:2007 \\
\hline & $\begin{array}{l}\text { Tablero contrachapado Clase } \\
\text { Técnica } 2 \text { (Ambiente húmedo) } \\
\text { Requiere tratamiento } \\
\text { superficial. }\end{array}$ & 2 & 2 & $\begin{array}{c}\text { UNE-EN } \\
636: 2012+\mathrm{A} 1: 2015\end{array}$ \\
\hline & $\begin{array}{l}\text { Tablero microlaminado LVL } \\
\text { Clase Técnica } 2 \\
\text { (Ambiente húmedo) } \\
\text { Requiere tratamiento superficial }\end{array}$ & 2 & 2 & $\begin{array}{c}\text { UNE-EN } \\
14279: 2007+A 1: 2009\end{array}$ \\
\hline & $\begin{array}{l}\text { Tableros de madera maciza } \\
\text { Clase Técnica SWP2 (Ambiente } \\
\text { húmedo) Requiere tratamiento } \\
\text { superficial }\end{array}$ & 2 & 2 & UNE-EN 12775:2001 \\
\hline $\begin{array}{l}\text { Entramado } \\
\text { estructural: vigas de } \\
\text { madera }\end{array}$ & $\begin{array}{l}\text { Madera aserrada estructural } \\
\text { (Madera maciza sección } \\
\text { rectangular) } \\
\text { Requiere tratamiento superficial }\end{array}$ & 2 & 2 & $\begin{array}{c}\text { UNE-EN } 14081- \\
1: 2016\end{array}$ \\
\hline
\end{tabular}




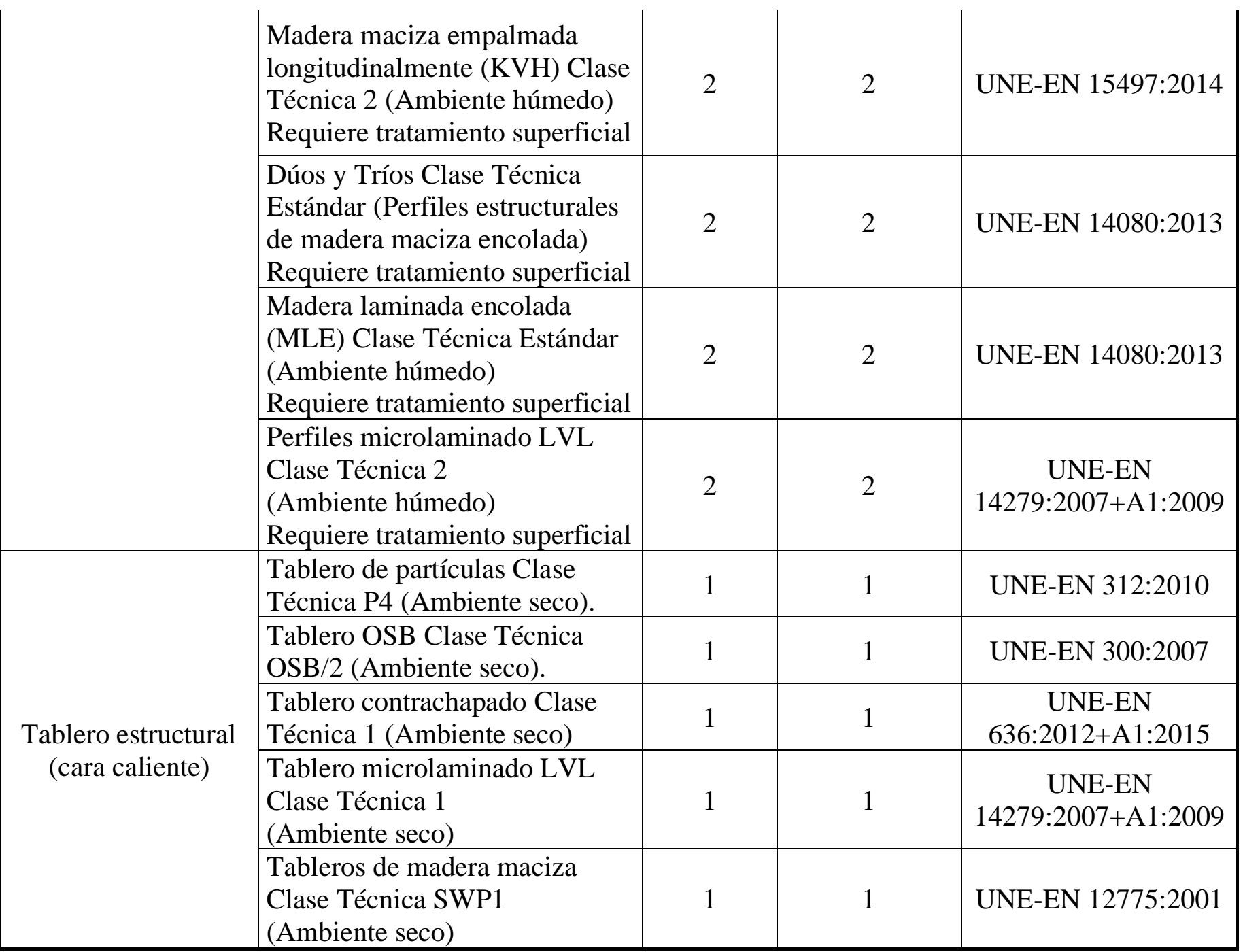

Tabla 13. Vinculación de productos de madera para cada elemento constructivo de madera de la solución constructiva FAP CLT - CVt.

\begin{tabular}{|c|l|c|c|}
\hline $\begin{array}{c}\text { ELEMENTO } \\
\text { CONSTRUCTIVO }\end{array}$ & \multicolumn{1}{|c|}{ PRODUCTOS } & $\begin{array}{c}\text { CLASE } \\
\text { DE } \\
\text { USO }\end{array}$ & $\begin{array}{c}\text { CLASE } \\
\text { DE } \\
\text { SERVICIO }\end{array}$ \\
\hline $\begin{array}{c}\text { Protección fachada: } \\
\text { paneles madera }\end{array}$ & $\begin{array}{l}\text { Madera aserrada de sección rectangular } \\
\text { (Ambiente exterior) } \\
\text { Requiere tratamiento en autoclave con protectores }\end{array}$ & 3 & 3 \\
\hline $\begin{array}{c}\text { Perfilería madera } \\
\text { (cara fría) }\end{array}$ & $\begin{array}{l}\text { Rastreles de madera } \\
\text { Requiere tratamiento en autoclave con protectores }\end{array}$ & 3 & 3 \\
\hline $\begin{array}{c}\text { Tablero estructural (cara } \\
\text { fría) }\end{array}$ & $\begin{array}{l}\text { Ver Productos para Tablero Estructural cara fría en CPT } \\
\text { NVSFC-ENT (Tabla 12) }\end{array}$ & - & - \\
\hline CLT & Madera contralaminada & 1 & 1 \\
\hline $\begin{array}{c}\text { Tablero estructural (cara } \\
\text { caliente) }\end{array}$ & $\begin{array}{l}\text { Ver Productos para Tablero Estructural cara caliente en } \\
\text { CPT NVSFC-ENT (Tabla 12) }\end{array}$ & - & - \\
\hline $\begin{array}{c}\text { Perfilería madera } \\
\text { (cara fría) }\end{array}$ & Rastreles de madera & 1 & 1 \\
\hline
\end{tabular}




\section{CONCLUSIONES}

Como conclusión de este trabajo se puede afirmar que la correcta utilización de la madera en construcción puede no resultar compleja para el proyectista si dispone de los datos necesarios para una correcta prescripción.

La durabilidad y la ausencia de patologías depende no solo de la elección de los materiales. En aquellos que estén más expuestos será necesario también contar con un diseño de soluciones que protejan a estos los materiales. Se deben cuidar los encuentros y los puntos singulares. Es decir, los diseños de las soluciones constructivas unido a una adecuada selección de productos de madera contribuyen a minimizar el riesgo de patologías y garantizar la durabilidad.

Para facilitar la prescripción se han propuesto un conjunto importante de soluciones tipificadas con vinculación a productos. Para ayudar en la búsqueda y selección de los productos se ha establecido un sistema de clasificación a la vez que se generará una herramienta de selección. La necesidad de datos hace referencia no solo a datos técnicos sino también a datos ambientales.

Los trabajos contenidos en el proyecto presentado no serán suficientes para abarcar toda la riqueza posible de soluciones, quedará pendiente una gran parte por evaluar siendo también necesario realizar ensayos de laboratorio.

\section{AGRADECIMIENTOS}

Los autores quieren agradecer al resto de miembros del Grupo Operativo los trabajos desarrollados durante el proyecto y en especial al Ministerio de Agricultura, Pesca y Alimentación del Gobierno de España y a la Unión Europea por la financiación dada al proyecto mediante el Programa Nacional de Desarrollo Rural 2014-2020, a través del Fondo Europeo Agrícola de Desarrollo Rural FEADER.

\section{REFERENCIAS}

Conde García, M., Castro-Nuño Cordero, D.F., Abad Garrido, B., Conde García, M., Fernández-Golfín Seco, J.I., Tenorio Ríos, J.A. (2017), Base de datos de valores ambientales de referencia en los productos de madera para la evaluación de la sostenibilidad en la construcción., Actas del $7^{\circ}$ Congreso Forestal. Plasencia.

Código Técnico de la Edificación. Ministerio de Fomento, España (http://www.codigotecnico.org). Fernández-Golfín, J.I.; Rivela, B.; Conde, M.; (2011) La sostenibilidad en los productos "técnicos" de madera para la construcción. Monografía Ache: Sostenibilidad y Construcción. 237-259. Barcelona. UNE-ENV 1995-1-1:1997. EUROCÓDIGO 5: Proyecto de estructuras de madera. Parte 1-1: Reglas generales y reglas para edificación.

UNE-EN 312:2010. Tableros de partículas. Especificaciones.

UNE-EN 300:2007. Tableros de virutas orientadas (OSB). Definiciones, clasificación y especificaciones.

UNE-EN 636:2012+A1:2015. Tableros contrachapados. Especificaciones.

UNE-EN 14279:2007+A1:2009. Madera microlaminada (LVL). Definiciones, clasificación y especificaciones.

UNE-EN 12775:2001. Tableros de madera maciza. Clasificación y terminología.

UNE-EN 14081-1:2016. Estructuras de madera. Madera estructural con sección transversal rectangular clasificada por su resistencia. Parte 1: Requisitos generales.

UNE-EN 15497:2014. Madera maciza estructural con empalmes por unión dentada. Requisitos de prestación y requisitos mínimos de fabricación.

UNE-EN 14080:2013. Estructuras de madera. Madera laminada encolada y madera maciza encolada. Requisitos. 
V1CC289CTE - CONPAT 2019, Tuxtla Gutiérrez, Chiapas, México

UNE-EN 14080:2013. Estructuras de madera. Madera laminada encolada y madera maciza encolada. Requisitos.

UNE-EN 14279:2007+A1:2009. Madera microlaminada (LVL). Definiciones, clasificación y especificaciones. 